\title{
ARTICLE \\ Chronic adolescent exposure to $\Delta 9$-tetrahydrocannabinol decreases NMDA current and extrasynaptic plasmalemmal density of NMDA GluN1 subunits in the prelimbic cortex of adult male mice
}

\author{
Virginia M. Pickel ${ }^{1}$, Faye Bourie ${ }^{1}$, June Chan $^{1}$, Ken Mackie $^{2}$, Diane A. Lane ${ }^{1}$ and Gang Wang ${ }^{1}$
}

\begin{abstract}
Adolescence is a vulnerable period of development when limbic connection of the prefrontal cortex (PFC) involved in emotional processing may be rendered dysfunctional by chronic exposure to delta-9-tetrahydrocannabinol $(\Delta 9-\mathrm{THC})$, the major psychoactive compound in marijuana. Cannabinoid-1 receptors (CB1Rs) largely mediate the central neural effects of $\triangle 9$-THC and endocannabinoids that regulate NMDA receptor-dependent synaptic plasticity of glutamatergic synapses in the prelimbic prefrontal cortex (PL-PFC). Thus, chronic occupancy of CB1Rs by $\triangle 9$-THC during adolescence may competitively decrease the functional expression and activity of NMDA receptors in the mature PL-PFC. We used a multidisciplinary approach to test this hypothesis in adult $\mathrm{C} 57 \mathrm{BL} / 6 \mathrm{~J}$ male mice that received vehicle or $\triangle 9-\mathrm{THC}$ in escalating doses (2.5-10 mg/kg/ip) through adolescence (postnatal day 29-43). In comparison with vehicle, the mice receiving $\triangle 9$-THC showed a hyperpolarized resting membrane potential, decreased spontaneous firing rate, increased current-induced firing threshold, and decreased depolarizing response to NMDA in deep-layer PL-PFC neurons analyzed by current-clamp recordings. Electron microscopic immunolabeling in the PL-PFC of adult mice that had received $\triangle 9$-THC only during adolescence showed a significant (1) decrease in the extrasynaptic plasmalemmal density of obligatory GluN1-NMDA subunits in dendrites of all sizes and (2) a shift from cytoplasmic to plasmalemmal distribution of GluN1 in large dendrites receiving mainly inhibitory-type synapses from CB1R-labeled terminals. From these results and concomitant behavioral studies, we conclude that social dysfunctions resulting from excessive intake of $\Delta 9$-THC in the increasingly available marijuana products used by male teens may largely reflect circuit defects in PL-PFC networks communicating through endocannabinoid-regulated NMDA receptors.
\end{abstract}

Neuropsychopharmacology (2020) 45:374-383; https://doi.org/10.1038/s41386-019-0466-9

\section{INTRODUCTION}

The psychoactive compound in marijuana, delta-9-tetrahydrocannabinol $(\triangle 9-\mathrm{THC})$, binds cannabinoid-1 receptors (CB1Rs) that are primary mediators of activity-dependent retrograde endocannabinoid signaling that decreases transmitter release [1]. CB1Rs are highly expressed in presynaptic terminals located in layers $2 / 3$ and 5 of the prefrontal cortex (PFC), where they regulate the synaptic strength and synaptic plasticity of glutamatergic inputs to pyramidal cells $[2,3]$. Chronic exposure to $\triangle 9$-THC during adolescence produces long-term behavioral dysfunctions that have some similarity to certain symptoms of psychiatric and neurodevelopmental disorders [4, 5], in which there is decreased functionality of NMDA receptors in the PFC [6].

NMDA receptors are ion channels whose functions are dependent on expression of GluN1 subunits [7]. At birth, GluN1 is expressed at low levels and rapidly increases throughout the early postnatal period to the third postnatal week coinciding with preadolescence $[8,9]$. GluN1 deletion in the prelimbic prefrontal cortex (PL-PFC) produces changes in social interactions and anxiety-like behaviors that have also been observed following chronic perinatal and adolescent administration of $\Delta 9$ THC $[10,11]$. The prominent role of CB1Rs in actomyosindependent cytoskeletal remodeling [4] further suggests that the neurons most affected by adolescent administration of $\triangle 9-T H C$ may have altered somatodendritic morphology and inputs from terminals containing CB1Rs. Thus, the behavioral abnormalities produced by chronic exposure to increasingly large quantities of $\triangle 9$-THC during adolescence may be concurrent with altered NMDA activity and GluN1 expression in PL-PFC neurons having distinct cytoarchitecture and afferent inputs. We examined this unexplored possibility using behavioral testing, current-clamp recording, and electron microscopic immunolabeling in the PLPFC of adult C57/BL6 male mice that received chronic escalating doses of $\triangle 9$-THC only through adolescence. This model has important implications for understanding the cellular mechanisms underlying behavioral effects produced by the heavy consumption of $\triangle 9-\mathrm{THC}$ by teenage males.

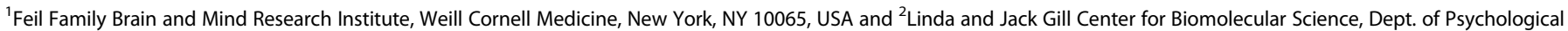
and Brain Sciences, Indiana University, Bloomington, IN 47404, USA
}

Correspondence: Virginia M. Pickel (vpickel@med.cornell.edu)

Received: 10 January 2019 Revised: 1 July 2019 Accepted: 12 July 2019

Published online: 19 July 2019 
a

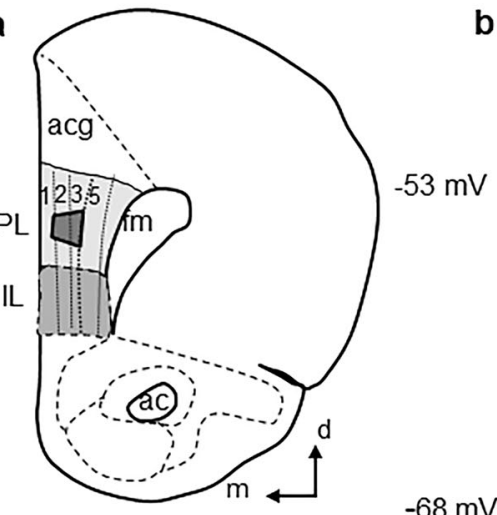

Adolescent Vehicle

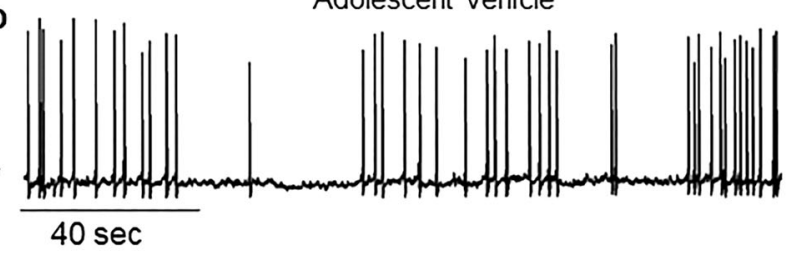

Current injected Adolescent $\triangle 9$-THC

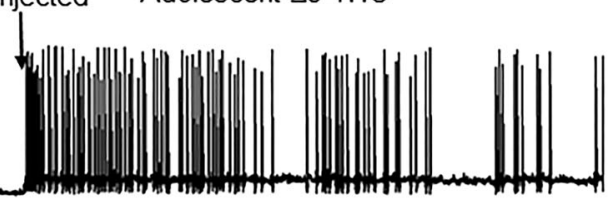

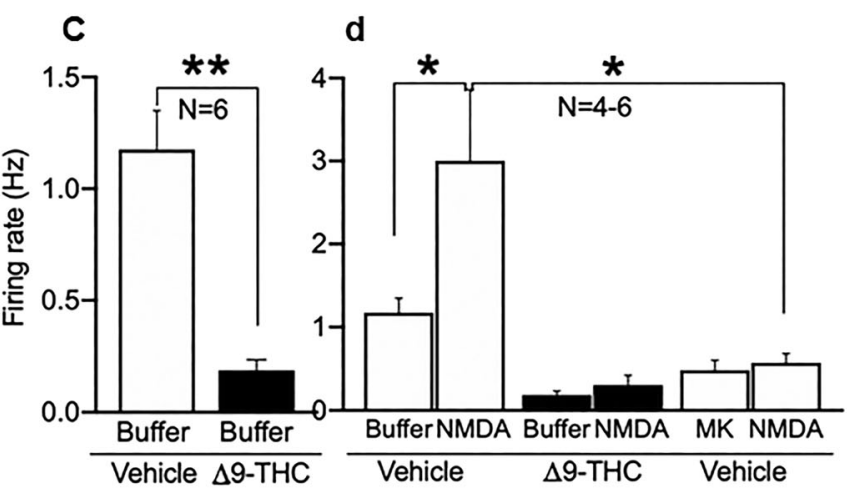

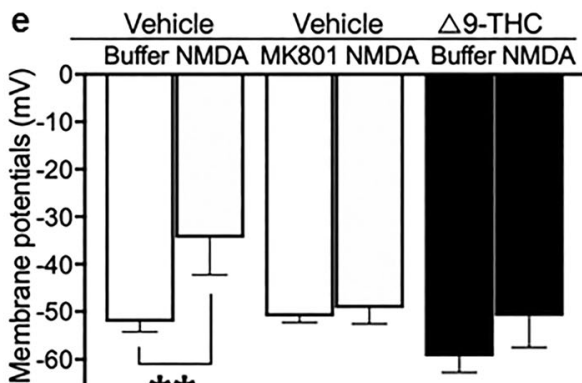

$\mathrm{N}=4-20$

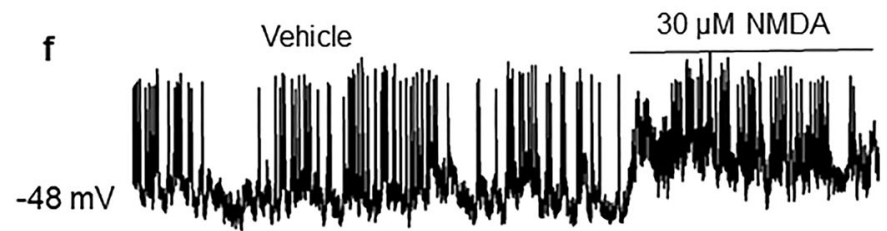

$\triangle 9$-THC vehicle

$30 \mu \mathrm{M}$ NMDA
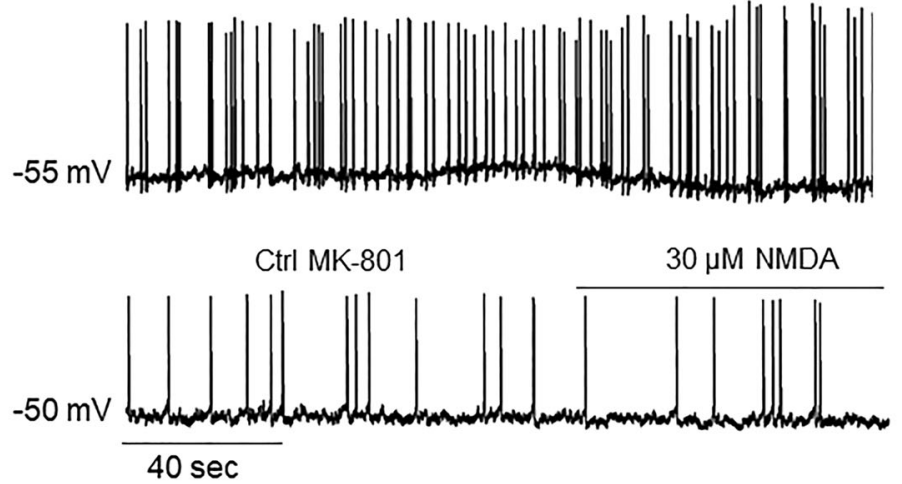

\section{MATERIALS AND METHODS}

Animals and drug treatment

All experimental procedures were carried out in accordance with the National Institutes of Health Guidelines for the Care and Use of Laboratory Animals and were approved by the Institutional Animal Care and Use Committees (IACUC) at Weill-Cornell Medical College. C57BL/6J male mice were obtained commercially from Jackson Laboratory (Bar Harbor, ME) immediately after weaning at postnatal day (PD) 21 and reared in groups of 4-5 mice/cage until reaching adulthood at PD70 [12]. All animals were kept in a temperature- and humidity-controlled environment, and maintained with HEPA-filtered air on a 12-h light/dark cycle (lights on from 7 a.m. to 7 p.m.). Food and water were available ad libitum.

An alcohol solution of $\triangle 9$-THC was obtained from the Drug Supply Program of the National Institute on Drug Abuse (Rockville, MD, USA). Nitrogen gas was used to evaporate the alcohol from this solution $[13,14]$. The $\Delta 9$-THC residue was solubilized by briefly heating at $<100{ }^{\circ} \mathrm{C}$ in $0.9 \% \mathrm{NaCl}$ (saline) solutions containing Tween-80 [15]. The vehicle (saline and Tween-80) was used to prepare $2.5,5.0$, and $10.0 \mathrm{mg} / \mathrm{kg}$ doses of $\Delta 9-T H C$, each of which was administered by once-daily intraperitoneal injections for 5 consecutive days from postnatal day (PD) 28 to 43, a period which corresponded to the later stage of adolescence in rodents [12]. 
Fig. 1 Adolescent exposure to $\triangle 9$-THC decreases activity of PL-PFC neurons. a Schematic diagram of the prelimbic (PL) region of the PFC, which was used for current-clamp recording in layer 5 and electron microscopic analysis (trapezoid) in layers $2 / 3$ of the PL-PFC. Other identified brain structures include anterior cingulate cortex (acg), infralimbic cortex (IL), forceps minor corpus callosum (fmi), and anterior commissure (ac). Arrows point medial $(\mathrm{m})$ and dorsal (d) in this modified drawing of a half coronal section at $1.98 \mathrm{~mm}$ anterior to Bregma as seen in the mouse brain atlas [19]. b Representative traces of the membrane potential and spontaneous discharge in patch-clamp recordings of deep layer PL-PFC neurons from adult mice receiving vehicle (upper trace) or $\Delta 9$-THC (lower trace) during adolescence. The spontaneous spikes seen in slices from vehicle-injected adolescent mice are absent in recordings from a PL-PFC neuron in a mouse receiving $\Delta 9$-THC in adolescence, which shows a firing pattern that is dependent on the injected current. c Bar graphs summarize the changes in spontaneous firing rate in PL-PFC neurons of the vehicle vs. the $\triangle 9$-THC groups (from $1.18 \pm 0.17 \mathrm{~Hz}$ of vehicle to $0.19 \pm 0.05 \mathrm{~Hz}$ of $\Delta 9-\mathrm{THC}, P<0.01, N=6$ ). d The presence of the NMDA receptor antagonist MK-801 $(10 \mu \mathrm{M})$ blocked NMDA $(30 \mu \mathrm{M})$-induced increase in firing rate (from $3.0 \mathrm{~Hz} \pm 0.86 \mathrm{~Hz}$ of vehicle to $0.57 \pm 0.11 \mathrm{~Hz}$ of $\Delta 9-\mathrm{THC}, P<0.05, N=4-5$ ). e Bar graphs summarize changes in RMP in PL-PFC neurons in vehicle compared with $\triangle 9$-THC-injected mice. NMDA-induced depolarization in mice receiving vehicle injections during adolescence is abolished in mice receiving $\triangle 9-\mathrm{THC}$ or in the presence of MK-801 (from $3.0 \pm 0.86 \mathrm{~Hz}$ of vehicle to $0.48 \pm 0.12 \mathrm{~Hz}$ of $\triangle 9-\mathrm{THC}, P<0.05, N=4-5$ ). The presence of the NMDA $(100 \mu \mathrm{M})$ induce depolarization in the vehicle groups (from $-52.2 \pm 2.1 \mathrm{mV}$ of vehicle to $-34.4 \pm 7.8 \mathrm{mV}$ of NMDA, $P<0.05, N=4-5$ ). However, there is no depolarization induced by NMDA in $\triangle 9$-THC or MK-801-treated groups $(P>0.05, N=4-12)$. $f$ Representative traces from PL-PFC neurons from mice receiving $\triangle 9$-THC (middle trace) does not result in depolarizing responses to NMDA as seen in mice receiving vehicle (upper trace). The presence of MK-801 inhibited the NMDA-induced increase in firing rate and depolarization (lower trace). $N=$ number of mice per treatment group. Unpaired $t$-test ${ }^{*} p<0.05$

The mice were assigned randomly to $\triangle 9$-THC- and vehicle treatment groups. After completion of the last injection on PD 43 , the mice were returned to their home cages without further injections while conducting behavioral tests in the 27-day interim from PD 43 to young adulthood at PD70.

\section{Whole-cell current-clamp recordings}

Whole-cell current-clamp recording was used to determine whether adolescent administration of $\triangle 9$-THC has a long-term effect on the electrophysiological activities in layer V of the PL-PFC $[2,16]$. For this, slice preparations of frontal cortical tissue from vehicle- $(n=6)$ and $\Delta 9$-THC-pretreated $(n=6)$ mice were obtained and stored in ice-cold sucrose-artificial cerebrospinal fluid (s-aCSF), and perfused with the $\mathrm{Mg}^{2+}$-free extracellular perfusion [17]. After the whole-cell configuration was formed, the access and membrane resistances were continuously monitored through the recording. Only those cells, in which access resistance was stable (change $<10 \%$ ), were included in the data analysis. Stable baseline recordings of the resting membrane potentials (RMP) were achieved before local application of the buffer control, NMDA, or MK-801 buffers. Major classes of pyramidal cells and interneurons were defined by their spontaneous firing patterns without or with an injected current $[17,18]$. Data are expressed as means \pm SEM in recorded cells from at least four mice per treatment group. The two-tailed unpaired $t$-test was used to compare the difference between the vehicle and drug-induced effect on the RMP and spontaneous firing rate. Statistically, $P<$ 0.05 and $P<0.01$ were considered significant and very significant, respectively. RMP and spontaneous firing were recorded using Window pClamp Clampex 10.3 and analyzed offline using Clampfit 10.3 (Molecular Devices).

\section{Electron microscopic immunolabeling}

Vehicle- and $\triangle 9$-THC-pretreated adult mice were deeply anesthetized by intraperitoneal injection of sodium pentobarbital $(150 \mathrm{mg} / \mathrm{kg})$, and subjected to sequential vascular perfusion with $3.75 \%$ acrolein and $2 \%$ paraformaldehyde in $0.1 \mathrm{M}$ phosphate buffer (PB; pH 7.4) saline. Coronal sections through the PL-PFC at $1.5-2.5 \mathrm{~mm}$ anterior to Bregma [19] were collected from the aldehyde fixed brains of mice in each treatment group $(n=10$ / group). These were processed using previously described methods for dual immunoperoxidase and immunogold-silver labeling $[20,21]$. For this, these sections were incubated for $24 \mathrm{~h}$ at room temperature in a solution containing mouse monoclonal GluN1 antibody (Pharmingen, San Diego, CA, USA) and guinea pig polyclonal CB1R affinity-purified antisera at the respective dilutions of 1:50 and 1:3200 in Tris-buffered saline (TS; $\mathrm{pH} 7.6$ ) and $0.1 \%$ bovine serum albumin (BSA; Sigma-Aldrich, St. Louis, MO).
The GluN1 antibody was directed against a fusion protein corresponding to amino acids $660-811$ of GluN1. Western blot analysis of the GluR1 antibody in hippocampal homogenates and rat synaptic membranes shows one major band at $116 \mathrm{kD}$ comparable with that seen in HEK293 cells transfected with cDNA encoding GluN1, which is absent in western blots of nontransfected cells [22]. This antibody has been extensively used for light and electron microscopy in rodent [23] and shown to be specific in mouse forebrain by absence of immunolabeling after spatial-temporal GluN1 gene deletion [23]. The CB1R was identified by an affinity-purified polyclonal antibody raised in guinea pig against a glutathione S-transferase fusion protein containing the $C$ terminus [residues $401-473$ of rat CB1R]. This antibody has been shown to have no immunoreactivity in CB1R knockout mice [24].

Following the incubation with primary antiserum, the tissue was incubated for $30 \mathrm{~min}$ in a TS and $0.1 \%$ BSA solution containing 1:200 dilution of donkey anti-guinea pig biotinylated lgG (Jackson Immunoresearch, West Grove, PA) then washed in TS and given a 30-min incubation in Vector ABC Elite kit (Vector Labs, Burlingame, $(\mathrm{A})$. The peroxidase reaction product was visualized by reaction in 3, 3'-diaminobenzadine (DAB, Sigma-Aldrich, St. Louis, MO) with $0.1 \%$ hydrogen peroxide. Subsequently the tissue-sections were (1) washed in TS, (2) incubated overnight in a 1:50 dilution of Ultrasmall gold conjugated to goat anti-mouse IgG. (Electron Microscopy Sciences, Hatfield, PA), and (3) silver-intensified in Amersham Silver intense kit (GE Biosciences, Piscataway, NJ). Reversal of markers and immunolabeling with omission of one or both primary and secondary antisera were used as controls for spurious labeling. The immunolabeled sections were rinsed in $0.2 \mathrm{M}$ citrate buffer and $0.1 \mathrm{M}$ PB prior to postfixation in $2 \%$ osmium tetroxide and embedding in Epoxy resin using conventional electron microscopic methods [20].

\section{Image analysis}

The electron microscopic analysis was conducted in ultrathin sections collected from the PL-PFC in two plastic-embedded vibratome sections from ten adult mice that received vehicle $(n=$ $5)$ or $\triangle 9$-THC $(n=5)$ during adolescence. The density of immunolabeled and unlabeled neuronal profiles was tabulated in a total surface area of $21,170 \mu^{2}$ collected equally from $\sim 70$ images from each of the ten mice. MCID Analysis software, Version 7.0 (Focus Ltd, Cambridge, UK) was used to determine the mean diameter of GluN1-labeled dendritic profiles. The immunolabeled dendritic profiles were statistically separated into small, medium, and large categories using cluster analysis. Differences in GluN1 density between drug treatment groups and dendritic size were analyzed with a two factor (drug $\times$ size) ANOVA using JMP 

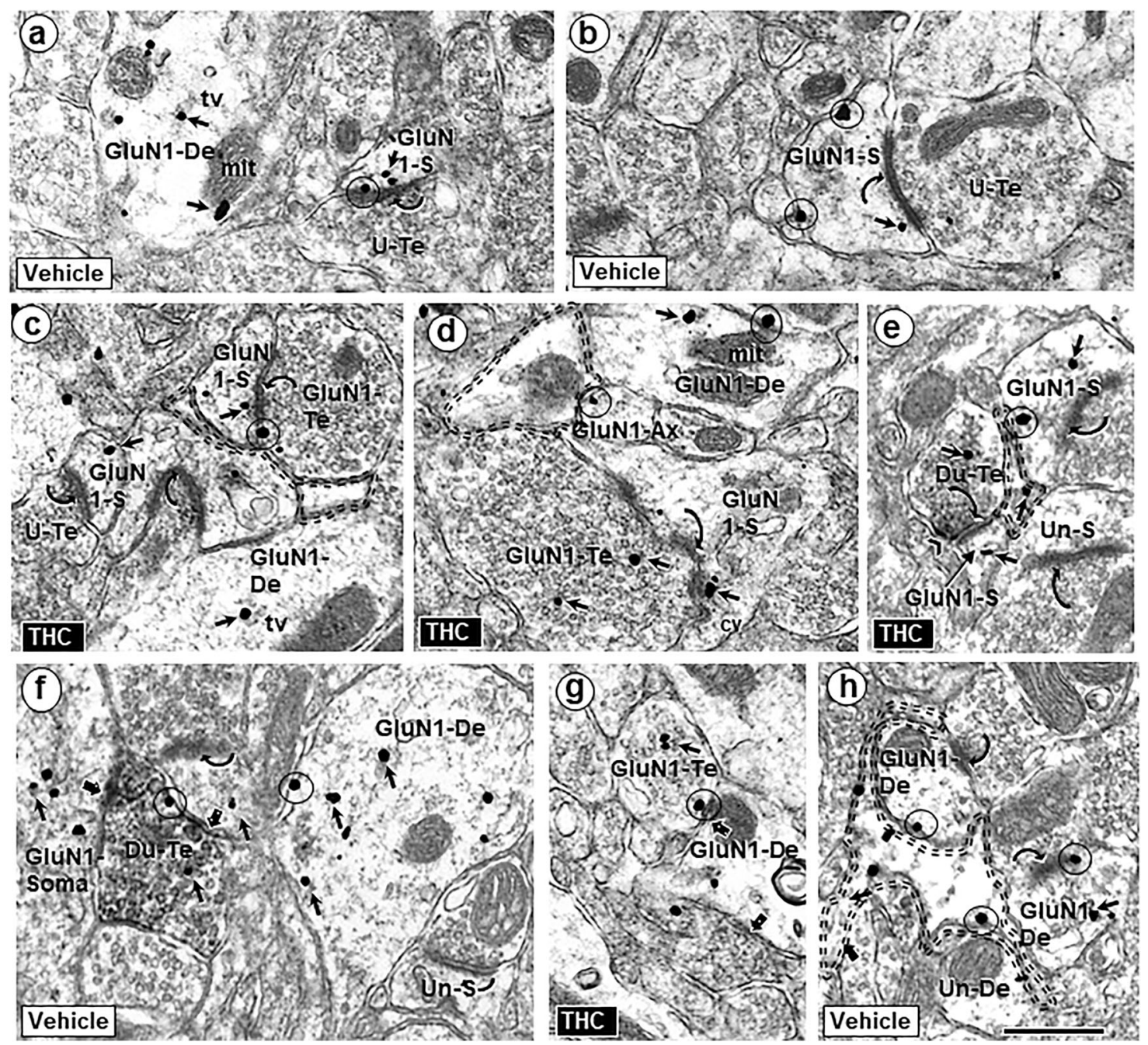

Fig. 2 Similarities of GluN1-immunogold distribution in neuronal profiles contacted by other neuronal and glial structures in the PL-PFC of adult mice that received vehicle or $\Delta 9-\mathrm{THC}$. GluN1 is seen within the cytoplasm (small arrows) and on plasma membranes (circles) of selective neuronal (dendritic and axonal) and perisynaptic glial profiles in the PL-PFC of adult mice that received vehicle (a, b) or $\Delta 9-\mathrm{THC}(\mathbf{c}, \mathbf{d})$ during adolescence. In these images GluN1-immunogold particles are seen in dendritic spines (GluN1-s) receiving asymmetric synapses (curved arrows) from unlabeled terminals or GluN1-labeled terminals. GluN1 immunogold is also respectively localized together (Du-Te) or separate (GluN1-Te) from dense CB1R-immunoperoxidase labeling in axon terminals forming symmetric synapses (block arrows) with GluN1-labeled somatodendritic profiles (GluN1 Soma) and dendrites (GluN1-De). e Dual labeled terminals (Du-te) contain sparse gold GluN1 and CB1-immunoperoxidase labeling (chevron). In $\mathbf{f}$, unlabeled axon terminals form asymmetric synapses (curved arrows) with a dendritic spine that is contacted by the dual labeled terminal (upper left) and an unlabeled dendritic spine (Un-s) opposed to the GluN1-De. tv = tubulovesicles (tv), mit = mitochondrion, $\mathrm{U}-\mathrm{Te}=$ unlabeled terminal; Un-S $=$ unlabeled spine; scale bar $=500 \mathrm{~nm}$. A GluN1-immunogold labeled dendrite (GluN1-De) is opposed to a GluN1-labeled terminal (GluN1-Te) and to a perisynaptic glial process (double dashed line) in $\mathbf{g}$ and $\mathbf{h}$, respectively

Statistical Discovery from SAS (Cary, NC). Chi-square analysis was used to determine the statistical significance of between group differences in dendritic spine density in the PL-PFC. Differences were considered significant at $p<0.05$.

Microscopic illustrations were prepared by importing digital images into Adobe Photoshop (CS4) and Powerpoint (Microsoft Office, 2016) to enhance contrast, prepare composite plates, and add lettering. Labeled neuronal and glial profiles were defined using the nomenclature of Peters et al. [25].

Behavioral testing

The following behavioral tests were conducted during the light phase of the light/dark cycle in a subgroup of young adult (PD70) mice that received vehicle $(n=4)$ or $\triangle 9$-THC $(n=3)$ from PD 28 to 43. The apparatus used for each behavioral task was cleaned thoroughly with $70 \% \mathrm{EtOH}$ between mice in order to remove olfactory cue biases. To avoid potential confounding effects of behavior testing, these tests were done in a group of mice separate from those used for current-clamp recording or electron microscopic immunolabeling.

Locomotor activity was analyzed for $30 \mathrm{~min}$ in standard mouse open-field chambers (Med Associates, St. Albans, VT) using infrared beam breaks to measure both horizontal movement and rearing [26]. The distance traveled $(\mathrm{cm})$, average speed $(\mathrm{cm} / \mathrm{s})$, stereotypic counts, and ambulatory time in the center vs. periphery were video recorded. Unpaired $t$-tests (SPSS IBM Corp, version 20) were conducted for between group comparisons of each of these parameters with a $p<0.05$ considered as statistical significant.

Social/Novel Object Interaction Test The conventional threechamber paradigm test for sociability and preference for social approach and novelty [27] was modified with the use of a rectangular corrugated plastic box $(27 \times 16.5 \times 9.25 \mathrm{in}$.) without 

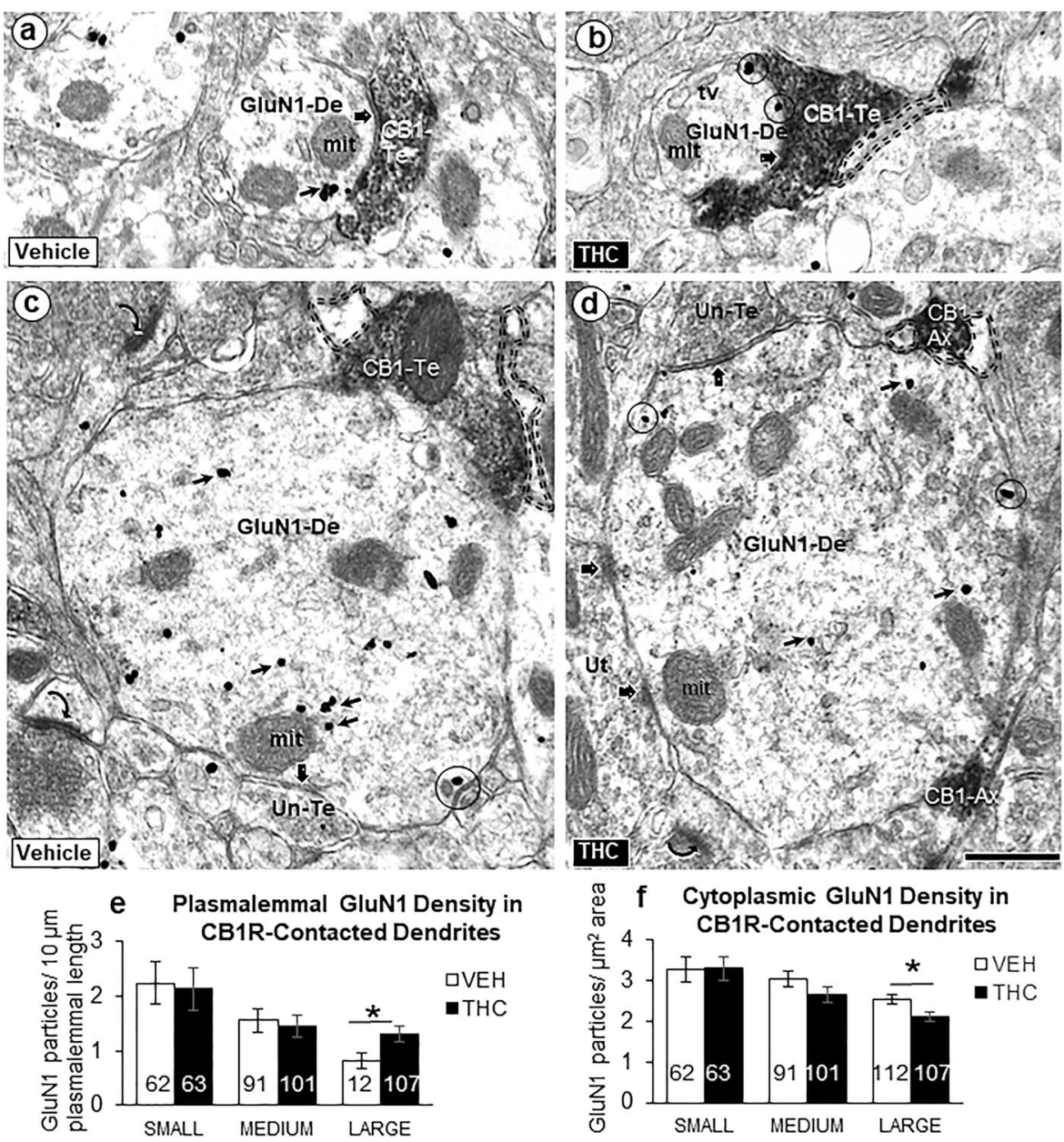

Fig. 3 Postsynaptic GluN1-immunogold labeling in dendrites receiving synaptic contacts from CB1R-labeled terminals. Electron microscopic images (a-d) and bar graphs (e, f) showing size-dependent changes in the plasmalemmal and cytoplasmic GluN1-immunogold distribution in dendrites recipient to inputs from small axons or terminals that are unlabeled or contain immunoperoxidase labeling for CB1Rs in PL-PFC of adult mice receiving $\triangle 9$-THC compared with vehicle. GluN1 immunogold is seen on the plasma membrane (circles) and in the cytoplasm (small arrows) of small $(\mathbf{a}, \mathbf{b})$ and large (c, $\mathbf{d})$ dendritic profiles (GluN1-De). These profiles receive symmetric synapses (block arrows) from axon terminals that are unlabeled (Un-Te) or contain dense immunoperoxidase labeling for the CB1R (CB1-Te). The GluN1-labeled dendrites are opposed to glial processes (profiles outlined with dashed line) in $\mathbf{b}-\mathbf{d}$ and to a CB1R-labeled axon (CB1-Ax) in d. Curved arrows = asymmetric excitatory-type axospinous synapses in the neuropil of panel c. Scale bar $=500 \mathrm{~nm}$. Bar graphs of e and $\mathbf{f}$ show the number of GluN1-labeled dendritic shafts in $21,170 \mu \mathrm{m}^{2}$ of PL-PFC tissue collected equally from $\sim 70$ microscopic images in two vibratome sections from five THC and five VEH-injected adult mice. ANOVA ${ }^{*} p<0.05$

dividing plastic walls. A metal wire was affixed across the top of the apparatus to separate the box into two equally-sized chambers. Each subject was given $5 \mathrm{~min}$ to acclimatize to the open box before being briefly placed back inside their home cage while a conspecific stranger mouse and an inanimate, novel object were placed in identical wire pencil cages on opposite chambers, respectively designated as those containing social (SOC) and novel object (NOV).

Mice were placed in the center of the box and given $10 \mathrm{~min}$ to explore the two chambers. ANY-maze software was used for video recording and tracking of each test to quantify the time spent and number of entries of the mouse into the respective SOC and NOV contact zones, which were defined by the perimeter of a 2-2.5-in. circle around the pencil cages. Two-way ANOVAs were used to assess main effects and potential interaction effects between treatment condition and zone(s). In the case that variances between groups were found to be significantly different $(p<0.05)$, a Welch's adjustment was made for the Levene's test for equality of variances.

Y-maze Spontaneous Alternation Task was used to assess spatial working memory and learning as previously described [22, 28].
The task involves testing the ability of the subject to remember a previously visited arm and takes advantage of the natural explorative drive of rodents. A lower spontaneous alternation (SA) score indicates reduced cognitive function. In this test, mice were placed in the center of the maze and given $10 \mathrm{~min}$ to freely explore each arm. An arm entry was recorded when $85 \%$ of a mouse's body entered the arm. The total number of arm entries and percentage of SAs were recorded using ANY-maze software (Stoelting, Wood Dale, IL). A SA occurred when a mouse entered into a different arm of the maze in each of three consecutive arm entries. The percentage of SA was calculated as the total number of alternations (i.e., number of entries into three different arms consecutively), divided by the total number of arm entries, multiplied by 100 .

\section{RESULTS}

Whole-cell current-clamp recording

Recordings from neurons located in deep layers of the PL-PFC (Fig. 1a) of adult mice showed that receipt of adolescent $\triangle 9$-THC during adolescence reduced glutamate activity (Fig. 1b), and 
Table 1. Area density of dendritic profiles with and without GluN1 labeling in the PL-PFC of adult mice chronically exposed to THC as adolescents

\begin{tabular}{llll}
\hline Profiles & Vehicle $(n)$ & THC $(n)$ & $\%$ Change in profiles \\
\hline Dendritic spines & & & \\
GluN1 positive & 202 & 296 & $\uparrow 46.5 \%$ \\
GluN1 negative & 1768 & 2283 & $\uparrow 29.1 \%$ \\
Column total & 1970 & 2579 & $\uparrow 30.9 \%^{*}$ \\
$\%$ GluN1 positive & $11 \%$ & $11 \%$ & $0 \%$ \\
Dendritic shafts & & & \\
GluN1 positive & 1581 & 1427 & $\downarrow 9.7 \%$ \\
GluN1 negative & 948 & 1053 & $\uparrow 11.1 \%$ \\
Column total & 2529 & 2480 & $\downarrow 1.9 \%$ \\
$\%$ GluN1 positive & $62.80 \%$ & $57.50 \%$ & $\downarrow 5 \% * *$ \\
\hline
\end{tabular}

$n=$ number of dendritic spines and shafts observed in $21,170 \mu \mathrm{m}^{2}$ of PLPFC tissue collected equally from $\sim 70$ microscopic images in two vibratome sections from adult mice receiving $\triangle 9$-THC $(n=5)$ or vehicle $(n=5)$ during adolescence. The receipt of $\Delta 9-T H C$ during adolescence resulted in a significant $\left(^{*}\right)$ increase in the density of dendritic spines as compared with mice receiving vehicle injections $\left(x^{2}(1)=81.51, p<0.0001\right)$ without changing the proportion of GluN1 immunolabeling between the two treatment groups $\left(x^{2}(1)=1.7^{2}, p>0.05\right)$. In contrast, there was no difference in the density of dendritic shafts between $\Delta 9$-THC-injected and vehicle-injected controls $\left(x^{2}(1)=0.43, p>0.05\right)$; however, there was a significant $\left(^{* *}\right)$ decrease in the number of GluN1-positive dendritic shafts $\left(x^{2}(1)=12.92, p, 0.0003\right)$

increased the threshold for spontaneous firing (Fig. 1c: $\triangle 9$-THC vs vehicle, $P<0.01, N=6)$. Bath application of NMDA $(30 \mu \mathrm{M})$ elicited depolarization in PL-PFC neurons from vehicle-injected mice, which was attenuated in recordings from mice receiving $\triangle 9$-THC (Fig. $1 \mathrm{~d}$ : control buffer vs. NMDA, $P<0.05, N=5-6$; vehicle vs. $\Delta 9$ THC, $P<0.01, N=4-5)$. Bath application of NMDA $(30 \mu \mathrm{M})$ also produced an increased firing rate in PL-PFL neurons from vehicleinjected animals (Fig. 1e: control buffer vs. NMDA, $P<0.05, N=$ 7-20), which was largely absent following bath application of NMDA receptor antagonist MK-801 $(10 \mu \mathrm{M})$ and in adult mice receiving $\triangle 9$-THC through adolescence (Fig. 1e: NMDA vs. MK-801, $P>0.05, N=4-6)$.

GluN1-immunogold distribution in dendritic and axonal profiles GluN1 immunogold was predominately seen in dendritic shafts and dendritic spines in the PL-PFC of adult mice receiving systemic injections of either $\triangle 9$-THC or vehicle during adolescence (Fig. 2). In dendritic shafts, the GluN1 labeling was most abundant in the cytoplasm and often localized to tubulovesicles and outer mitochondrial membranes (Fig. 2a, d). GluN1-immunogold particles were also seen on synaptic and extrasynaptic plasmalemmas of varying sized dendritic spines that received asymmetric excitatory-type synapses from GluN1-labeled and unlabeled terminals in the PL-PFC of both vehicle and $\triangle 9$-THC recipient mice (Fig. 2). Larger dendritic spines in each treatment group frequently displayed in-folded, discontinuous postsynaptic membrane specializations that were decorated with GluN1 immunogold (Fig. 3a-d).

Unlabeled (Fig. 2a, b), GluN1-labeled (Fig. 2c, d), and dual GluN1/CB1R-labeled axon terminals (Fig. 2e) formed asymmetric excitatory-type synapses mainly on dendritic spines. In these terminals, the immunogold particles were either distributed over synaptic vesicles or localized to presynaptic or extrasynaptic plasmalemmas (Fig. 2c, d). Axon terminals forming symmetric inhibitory-type synapses showed a similar subcellular distribution of GluN1, but more often contained only CB1R immunoreactivity
Table 2. Area density of GluN1-positive and -negative axon terminals forming asymmetric synapses with dendritic spines in the PL-PFC of adult mice exposed to adolescent vehicle or THC

\begin{tabular}{llll}
\hline Axon terminals & Vehicle $(n)$ & THC $(n)$ & $\%$ Change \\
\hline GluN1 positive & 183 & 234 & $\uparrow 27.9 \%$ \\
GluN1 negative & 1052 & 1017 & $\downarrow 3.3 \%$ \\
Column total & 1235 & 1251 & $\uparrow 1.2 \%$ \\
$\%$ GluN1 positive & $14.80 \%$ & $18.70 \%$ & $\uparrow 3.9 \%$ \\
\hline$n=$ number of axospinous synapses seen in $7978 \mu \mathrm{m}^{2}$ from THC and 8179 \\
$\mu \mathrm{m}^{2}$ in vehicle recipient mice
\end{tabular}

and preferentially contacted unlabeled and GluN1-labeled dendritic profiles (Fig. 2f, g). Astrocyte-like glial processes [25] frequently occupied portions of dendritic and axonal plasmalemmas without recognizable synaptic input in the PL-PFC of adult mice in both treatment groups (Fig. 2c-e and h).

Impact of adolescent $\Delta 9-\mathrm{THC}$ on synaptic and extrasynaptic GluN1 distribution

Receipt of $\triangle 9$-THC during adolescence substantially increased the number of asymmetric axospinous synapses on dendritic spines, the majority of which contained GluN1 immunogold (Table 1). Most of the presynaptic axon terminals at axospinous synapses also contained GluN1 immunogold (Table 2). In contrast with dendritic spines, there was a slight reduction in GluN1-positive and increase in GluN1-negative dendritic shafts receiving excitatory-type synapses in PL-PFC of $\triangle 9-T H C$ recipient mice (Table 1).

Administration of $\triangle 9$-THC during adolescence resulted in a qualitative increase in plasmalemmal and a decrease in cytoplasmic GluN1-immunogold particles in large, but not smaller dendritic profiles that received symmetric synapses from CB1Rlabeled terminals in adult PL-PFC (Fig. 3a-d). Statistical analysis confirmed a significant shift from cytoplasmic to plasmalemmal distribution of GluN1 in large dendrites that received synaptic input from axon terminals in the PL-PFC of $\triangle 9$-THC recipient mice (Fig. 3e, f).

GluN1-immunogold density on extrasynaptic plasmalemmas of all dendritic profiles was significantly reduced in the PL-PFC of adult mice that received $\triangle 9$-THC compared with vehicle during adolescence (Figs. 4 and $5 \mathrm{a}, \mathrm{b}$ ). The diagrams in Fig. 5c, d respectively show GluN1-immunogold distribution in PL-PFC dendritic profiles of varying sizes from vehicle- and $\triangle 9$-THCpretreated mice. Both synaptic (left) and extrasynaptic (right) distributions are represented in the two halves of each bisected dendritic segment.

Behavioral changes in adult mice receiving $\triangle 9-\mathrm{THC}$ during adolescence

Locomotor activity of adult mice receiving $\triangle 9$-THC during adolescence did not significantly differ $(t$-test, $p>0.05)$ from the vehicle controls in the open field or in chambers used for social/ novel object testing in which there were no treatment-specific differences in total distance traveled $(t(5)=0.99, p=0.37)$ or average speed $(t(5)=0.99, p=0.37)$ within the apparatus. There was also no significant interaction between drug treatment and compartment (ambulatory time: $(F(1,10)=3.77, p=0.081$ ); number of entries: $(F(1,10)=3.82, p=0.079)$.

There was a statistically significant effect for ambulatory time: $(F(1,10)=12.07, p=0.006)$ and number of entries: $(F(1,10)=11.03$, $p=0.008)$ for the interaction between treatment and contact zone (Fig. 5e, f). There was also a significant main effect of contact zone on ambulatory time $(F(1,10)=9.73, p=0.011)$ and number of entries $(F(1,10)=12.84, p=0.005)$. On average, mice receiving 

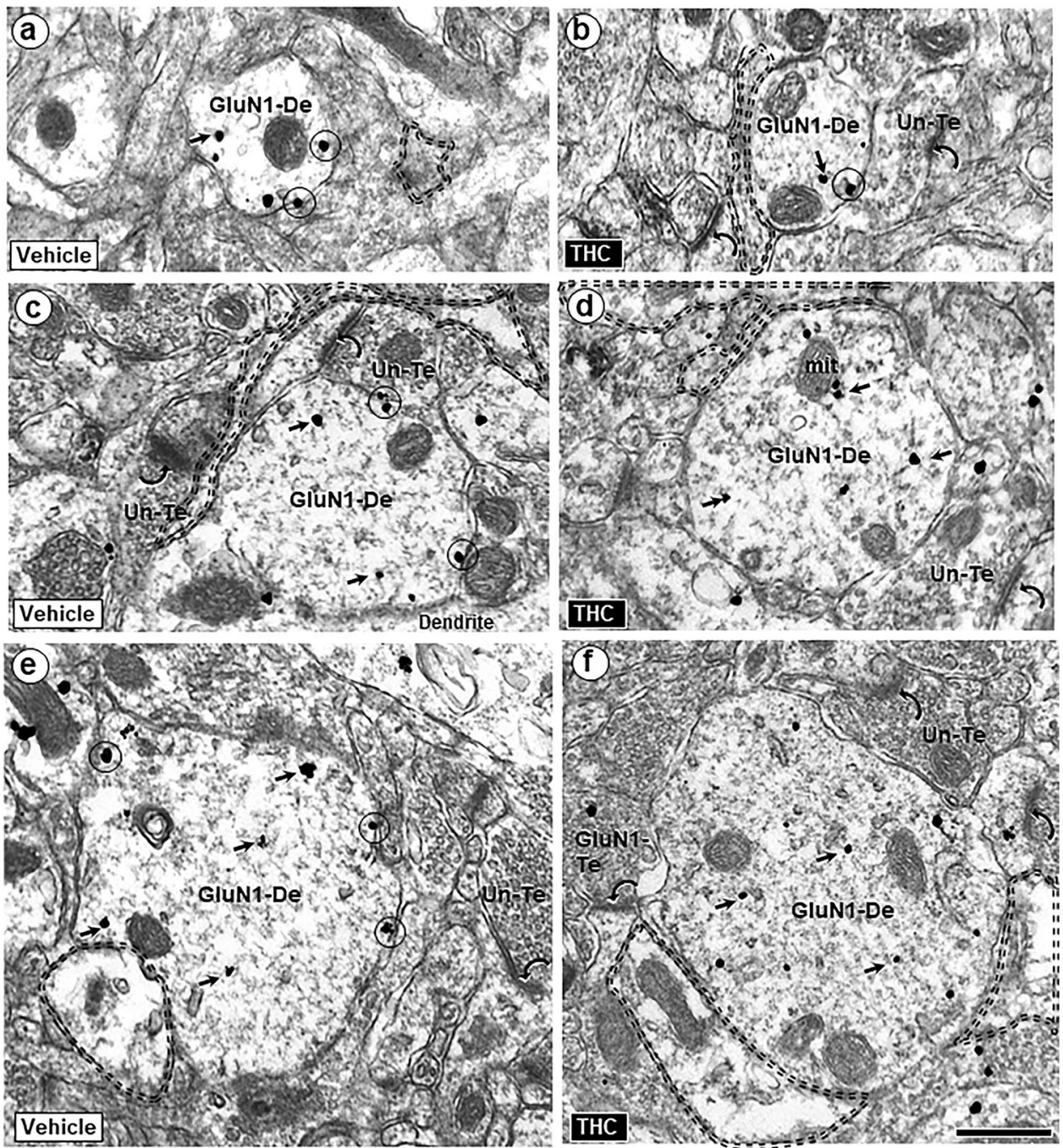

Fig. 4 Extrasynaptic GluN1-immunogold labeling in dendritic shafts in PL-PFC adult mice receiving vehicle or $\Delta 9$-THC during adolescence. The gold particles are located on the plasma membrane (circles) and in the cytoplasm (small arrows) of dendritic shafts (GluN1-De) having small $(\mathbf{a}, \mathbf{b})$, medium (c, d), and large $(\mathbf{e}, \mathbf{f})$ mean diameters. Many of these dendrites are opposed to glial processes (profiles outlined with dashed line) and to unlabeled (Un-Te) or GluN1-immunogold-labeled terminals (GluN1-Te) forming asymmetric synapses (curved arrows) on nearby dendritic spines. Scale bar $=500 \mathrm{~nm}$

$\triangle 9$-THC as adolescents spent significantly more time in the SOC contact zone $(258.43 \pm 1.77)$ compared with controls $(182.83 \pm$ 15.30). They also made significantly less entries into the NOV contact zone $(41 \pm 4.62)$ compared with vehicle-treated mice $(55.5 \pm 2.99)$ (Fig. $5 f$ and track plots in Fig. $5 \mathrm{~g}$ ).

When assessed for spatial working memory in the Y-maze, adolescent vehicle and $\triangle 9$-THC-treated mice performed similarly. Treatment groups did not differ significantly in the average percentage of spontaneous alternations made when tested as adults $(t(5)=0.184, p=0.86)$. Mice that received vehicle and $\Delta 9$ THC during adolescence also showed no significant differences in measures of locomotor activity, as reflected in ambulatory distance traveled $(t(5)=0.25, p=0.82)$, stereotypic counts $(t(5)=0.07, p=0.95)$, or ambulatory episodes average speed $(t(5)=0.36, p=0.73)$.

\section{DISCUSSION}

Our results provides new evidence that the firing pattern of deep-layer PL-PFC neurons in vehicle-injected mice is similar to that previously described in adult rats [29] and markedly disrupted by chronic adolescent administration of $\triangle 9-T H C$. The electrophysiological evidence for NMDA receptor hypofunction in these neurons is consistent with the observed decrease in extrasynaptic plasmalemmal density of GluN1 in dendritic profiles of all sizes in medial layers of the PL-PFC, which contain apical dendrites of many deep layer neurons. However, adolescent administration of $\triangle 9$-THC also produces a shift in GluN1 distribution from cytoplasmic to plasmalemmal sites in larger dendritic profiles that are major targets of inhibitory terminals that express CB1Rs [24]. These results together with the observed changes in number of axospinous synapses in the PL-PFC of adult mice that received $\triangle 9$-THC during adolescence suggest a lasting impact of adolescent $\triangle 9$-THC on the emergence of corticolimbic networks controlling adult behaviors as discussed below.

Adult male mice receiving $\triangle 9$-THC during adolescence have a higher density of GluN1-labeled axospinous synapses in PL-PFC We observed an increase in excitatory-type axospinous synapses in the PL-PFC of adult male mice receiving repeated injections of $\triangle 9-T H C$ in an escalating dose schedule similar to that described by Rubino et al. [16]. In this earlier study, adolescent administration of $\triangle 9$-THC decreased CB1R and CB1/G-protein activity in hippocampus and amygdala, but not in the PFC of male rats. The 

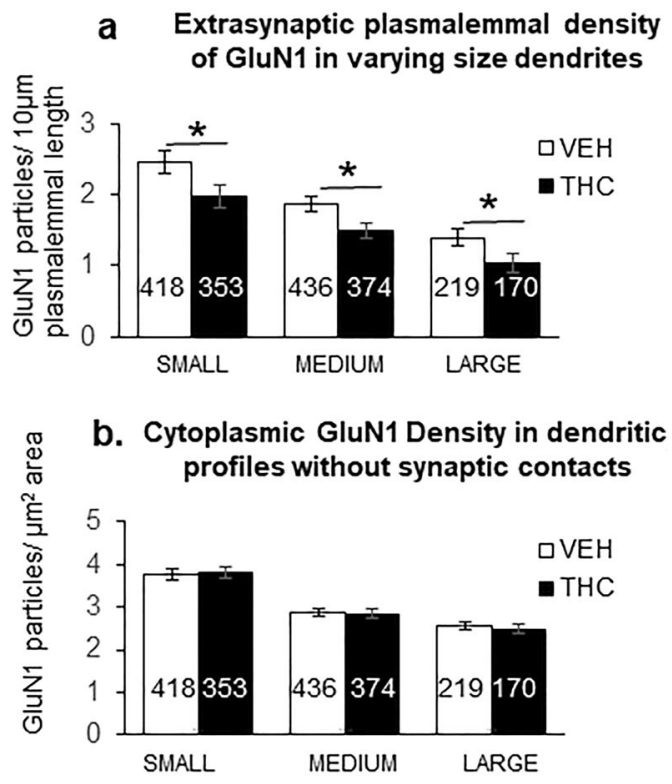

c-d. Summary of treatment-specific changes in dendritic GluN1 distribution

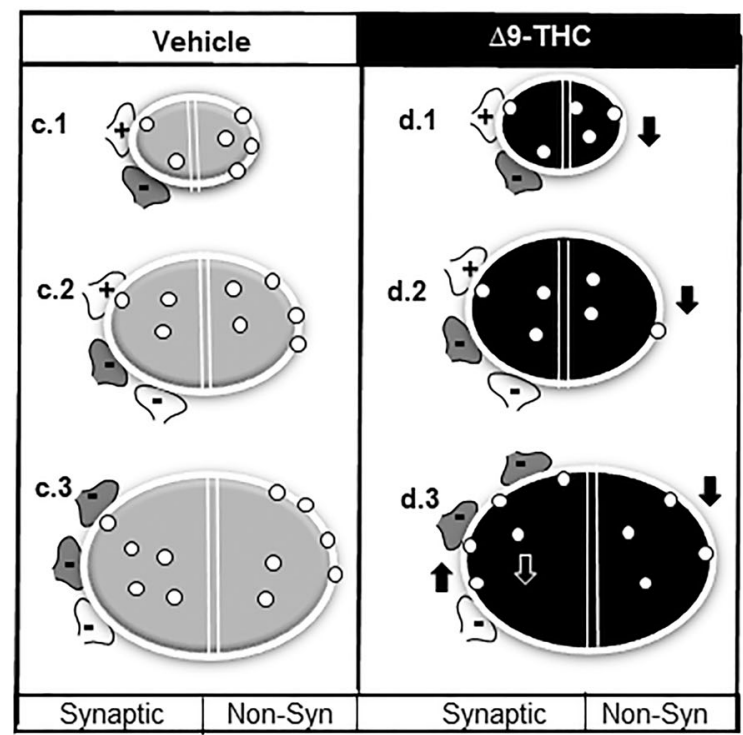

e. Time in contact zone with stranger mouse (SOC) and novel object (NOV)

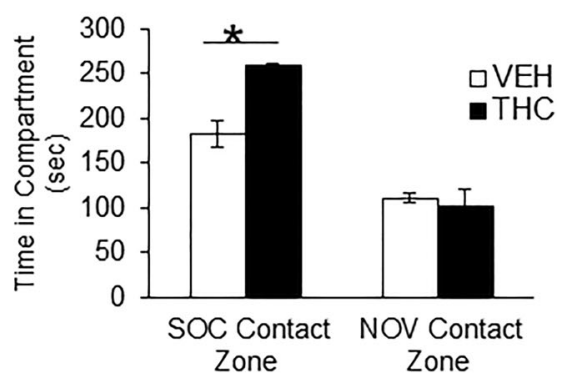

\section{f. Entries into contact zone}

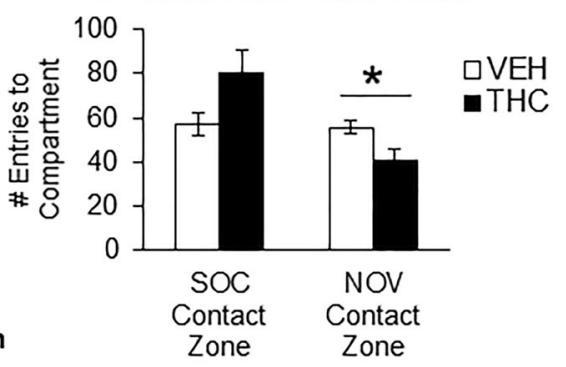

g. Track plots of mouse activity

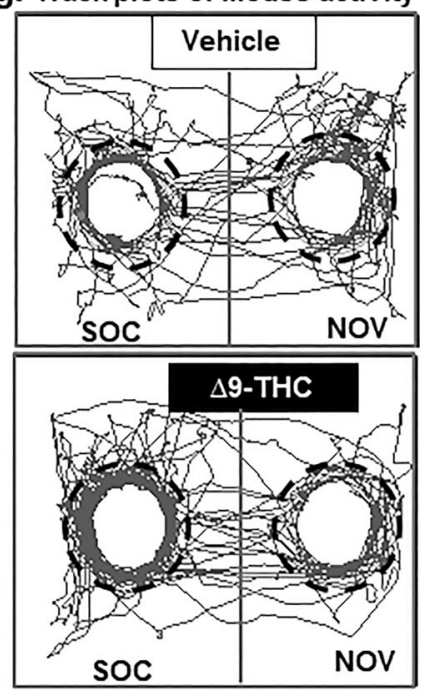

Fig. 5 Adult mice chronically exposed to $\triangle 9-T H C$ during adolescents differ from vehicle controls in their extrasynaptic GluN1 distribution in varying sized dendrites within the PL-PFC (a-d) and interactions with a stranger mouse (SOC) vs. novel object (NOV) (e-g). In a and $\mathbf{b}$, the bar graphs respectively show that adolescent $\triangle 9-\mathrm{THC}$ produces a significant decrease in extrasynaptic plasmalemmal GluN1 in small, medium and large dendrites, without effect on GluN1 cytoplasmic distribution in the PL-PFC dendrites of adult mice. In a and $\mathbf{b}$, ANOVA * $p<0.05$ and numbers = number of GluN1-labeled dendrites. Schematic drawings in $\mathbf{c}$ and $\mathbf{d}$ respectively summarize the GluN1-immunogold distribution in PL-PFC dendrites of adult mice receiving $\triangle 9$-THC or vehicle during adolescents. In each treatment group, transverse sections through small (c.1), medium (c.2), and large (c.3) dendrites are represented by oval shapes with a white line drawn around the perimeter to indicate the plasmalemma that encloses the cytoplasmic compartment. Each of these dendritic profiles is bisected by a double line to separate portions that are contacted by excitatory $(+)$ and inhibitory $(-)$ axon terminals (i.e., synaptic) or without axonal contact (i.e., non-synaptic (non-Syn)). CB1R labeling is shown by dark shading in a subgroup of the inhibitory-type terminals. Data from bar graphs in Fig. $3 e$, $f$ are schematically shown by an increase (up arrow) in plasmalemmal density and the corresponding decrease (down white arrow) in the cytoplasmic density of GluN1-immunogold particles (small circles) exclusively in large dendrites of mice receiving $\Delta 9$-THC compared with vehicle. In contrast, data summarized from bar graphs in this figure show a decrease (down arrow) in GluN1-immunogold density on the extrasynaptic plasma membrane of all dendrites without synaptic contact (i.e., non-synaptic). Bar graphs in $\mathbf{e}$ and $\mathbf{f}$ respectively show that administration of $\Delta 9$-THC compared with vehicle during adolescence significantly increases the time spent in portions of the chamber in contact with a stranger mouse and decreases the entries into the contact zone surrounding a novel object. ANOVA shows a statistically significant treatment effect on (ambulatory time: $(F(1,10)=12.07, p=0.006)$ and number of entries: $(F(1,10)=11.03, p=0.008)$. g Representative tracking of adult mice that received vehicle or $\triangle 9$-THC during adolescence shows that $\triangle 9$-THC enhances the mobilization to portions of the two interconnected compartments within which dashed lines indicate the perimeter of the contact zone around the stranger mouse (SOC) and novel object (NOV) 
downregulation of CB1R signaling in GABAergic terminals presynaptic to glutamatergic output neurons in amygdala and hippocampus, which project extensively to the PL-PFC [30] may serve to maintain less active dendritic spines normally eliminated during the protracted frontal cortical maturation [31]. The selective formation of dendritic spines contacted by axon terminals expressing presynaptic NMDA receptors and/or elimination of spines contacted by axon terminals lacking these receptors provides an equally plausible explanation for the observed increase in number of dendritic spines in the PL-PFC (see refs. $[32,33]$ for review). The glial profiles contacting excitatory-type synapse expressing pre-and/or postsynaptic GluN1 may represent sites of relevance to glial control of NMDA receptor-mediated glutamatergic transmission [34].

The notable increase in axonal distribution of GluN1 following chronic $\triangle 9$-THC activation of CB1Rs may reflect diminish endocannabinoid signaling that is implicated in axonal outgrowth and GluN1 polarization in cortical pyramidal cells [35]. The present detection of GluN1 in axon terminals in the PL-PFC of adult mice adds to the growing evidence that GluN1/GluN2B containing NMDA receptors are presynaptic autoreceptors and heteroreceptors in the mature as well as in the developing neocortex [36]. Many of these terminals form asymmetric excitatory-type synapses with dendritic spines and rarely express detectable levels of CB1R immunoreactivity, which is most prevalent in inhibitory-type terminals, some of which also contain GluN1 immunogold. In dually labeled terminals, CB1Rmediated retrograde signaling may oppose the NMDA-evoked release of GABA [37].

Adolescent $\triangle 9$-THC increases plasmalemmal density and decreased cytoplasmic GluN1 immunogold in large dendrites recipient to $\mathrm{CB} 1 \mathrm{R}$ terminals

Competitive disruption of CB1R-mediated endocannabinoid signaling may largely account for the observed increase in plasmalemmal and corresponding decrease in cytoplasmic GluN1 in PL-PFC dendrites of adults chronically exposed to $\Delta 9-T H C$ during adolescence. The large size of the affected dendrites is consistent with the known prevalence of CB1R terminals mainly on larger somatodendritic profiles of pyramidal neurons in the cerebral cortex [38].

The cytoplasmic and plasmalemmal distribution of GluN1 immunogold in dendrites of the PL-PFC confirms and extends earlier studies of GluN1 distribution in normal rodents [39, 40]. Within the cytoplasm, we often detected GluN1 immunogold along membranes of tubulovesicles and outer mitochondrial membranes, which play a key role in regulation of $\mathrm{Ca}^{2+}$ dynamics as well as in the intracellular trafficking, recycling, and degradation of GluN1 [40, 41]. The availability of a cytoplasmic pool of GluN1 subunits on these intracellular membranes may facilitate the regulation of NMDA receptor-mediated glutamatergic transmission in accordance with ongoing activity and stage of development $[42,43]$.

Repeated $\triangle 9$-THC administration during adolescence decreases extrasynaptic plasmalemmal density of GluN1 and NMDA current in PL-PFC neurons of adult mice

There was a significant reduction in GluN1 plasmalemmal and cytoplasmic density in large and the plasmalemma density in small and medium sized dendrites without synaptic contact in the PL-PFC of adult mice chronically exposed to $\triangle 9-\mathrm{THC}$ as adolescence. This suggests that as previously demonstrate for the developmentally regulated GluN2 subunits [7] GluN1 is internalized and internally sorted through degradative pathways. Our data further indicate that during the sensitive period of adolescent development chronic exposure to $\triangle 9$-THC may promote the activation of extrasynaptic NMDA receptors resulting from increased glutamate release. This possibility is supported by our results showing that many of the dendritic profiles without synaptic contacts are opposed to glial processes and/or neighboring excitatory-type axon terminals that are potential sources of glutamate and glycine, a co-agonist of extrasynaptic NMDA receptors [44], which inhibits surface trafficking of NMDA receptors [29].

Adolescent exposure to $\triangle 9-\mathrm{THC}$ during adolescence produces behavioral changes aligned with NMDA plasticity in PL-PFC neurons

Adult male mice chronically exposed to increasingly high doses of $\triangle 9-T H C$ during later stages of adolescence spent significantly more time and tended to have more entries into the contact zone around a conspecific stranger mouse. We also observed a significant decrease in NMDA current and plasmalemmal expression of GluN1 in PL-PFC neurons in separate subgroups of adult mice that received adolescent injections of $\triangle 9-\mathrm{THC}$ in a paradigm identical to the one used for testing social interactions. Together these results suggest that exposure to escalating doses of $\Delta 9-\mathrm{THC}$ during late adolescence increases preference for social novelty in adult mice through mechanisms involving downregulation of functional NMDA receptors in PL-PFC, which is similar to that produced by spatial-temporal GluN1 deletion in the medial PFC [45]. However, additional behavioral testing is needed to exclude other possibilities such as decreased social anxiety that also may be influenced by decreased NMDA receptor signaling in output neurons within the PFC [46].

As opposed to the increase in social interactions in $\Delta 9$-THCpretreated mice, these mice made significantly fewer entries into the contact zone around a novel object without any notable effect on the duration of time spent in this zone. This suggests that adolescent $\triangle 9$-THC may decrease exploratory behavior outside of social context, which provides sensory cues that promote lingering in the contact zone.

We observed no significant effect of adolescent administered $\triangle 9$-THC on measure of spatial working memory or spontaneous alternations in Y-maze testing of a subgroup of adult mice with identical drug treatment to those showing decreased NMDA GluN1 expression in PL-PFC neurons. The absence of effect on these tasks by a drug treatment that reduced glutamate activity in the PL-PFC is consistent with the know limited effect of cytotoxic PFC lesions on memory-related behaviors in adult mice [47]. These findings contribute to the emerging evidence that the complex cognitive and social behaviors are differentially dependent on output from prefrontal cortical and hippocampal networks [48]. However, there remains the possibility that subtle impairment of cognitive functions in mice receiving adolescent $\Delta 9$-THC may have been unrecognized because of the relatively small sample size in our study.

\section{FUNDING AND DISCLOSURE}

This research was supported by NIDA funding DA042943 to VMP and DA043982 to KM. $\triangle 9$-THC was provided by the Drug Supply Program of the National Institute on Drug Abuse (Bethesda, MD, USA). The authors declare no competing interests.

\section{ADDITIONAL INFORMATION}

Publisher's note: Springer Nature remains neutral with regard to jurisdictional claims in published maps and institutional affiliations.

\section{REFERENCES}

1. Hashimotodani $Y$, Ohno-Shosaku T, Kano M. Endocannabinoids and synaptic function in the CNS. Neuroscientist. 2007;13:127-37.

2. Fortin DA, Levine ES. Differential effects of endocannabinoids on glutamatergic and GABAergic inputs to layer 5 pyramidal neurons. Cereb Cortex. 2007;17: 163-74. 
3. Auclair N, Otani S, Soubrie P, Crepel F. Cannabinoids modulate synaptic strength and plasticity at glutamatergic synapses of rat prefrontal cortex pyramidal neurons. J Neurophysiol. 2000;83:3287-93.

4. Murphy $M$, Mills $S$, Winstone J, Leishman $E$, Wager-Miller J, Bradshaw $H$, et al. Chronic adolescent delta(9)-tetrahydrocannabinol treatment of male mice leads to long-term cognitive and behavioral dysfunction, which are prevented by concurrent cannabidiol treatment. Cannabis Cannabinoid Res. 2017;2:235-46.

5. Dow-Edwards D, Silva L. Endocannabinoids in brain plasticity: cortical maturation, HPA axis function and behavior. Brain Res 2017;1654(Pt B):157-64.

6. Catts VS, Lai YL, Weickert CS, Weickert TW, Catts SV. A quantitative review of the postmortem evidence for decreased cortical N-methyl-D-aspartate receptor expression levels in schizophrenia: how can we link molecular abnormalities to mismatch negativity deficits? Biol Psychol. 2016;116:57-67.

7. Sanz-Clemente A, Nicoll RA, Roche KW. Diversity in NMDA receptor composition many regulators, many consequences. Neuroscientist. 2013;19:62-75.

8. Luo J, Bosy T, Wang Y, Yasuda R, Wolfe B. Ontogeny of NMDA R1 subunit protein expression in five regions of rat brain. Dev Brain Res. 1996;92:10-17.

9. Sircar R. Developmental maturation of the N-methyl-D-aspartic acid receptor channel complex in postnatal rat brain. Int J Dev Neurosci. 2000;18:121-31.

10. Newsom RJ, Kelly SJ. Perinatal delta-9-tetrahydrocannabinol exposure disrupts social and open field behavior in adult male rats. Neurotoxicol Teratol. 2008;30:213-9.

11. Rompala GR, Zsiros V, Zhang S, Kolata SM, Nakazawa K. Contribution of NMDA receptor hypofunction in prefrontal and cortical excitatory neurons to schizophrenia-like phenotypes. PLoS ONE. 2013;8:e61278.

12. Spear LP. The adolescent brain and age-related behavioral manifestations. Neurosci Biobehav Rev. 2000;24:417-63.

13. Grim TW, Morales AJ, Thomas BF, Wiley JL, Endres GW, Negus SS, et al. Apparent CB1 receptor rimonabant affinity estimates: combination with THC and synthetic cannabinoids in the mouse in vivo triad model. J Pharm Exp Ther. 2017;362:210-18.

14. Goonawardena AV, Riedel G, Hampson RE. Cannabinoids alter spontaneous firing, bursting, and cell synchrony of hippocampal principal cells. Hippocampus. 2011;21:520-31.

15. Burston JJ, Wiley JL, Craig AA, Selley DE, Sim-Selley LJ. Regional enhancement of cannabinoid $\mathrm{CB}_{1}$; receptor desensitization in female adolescent rats following repeated delta-tetrahydrocannabinol exposure. Br J Pharm. 2010;161:103-12.

16. Rubino T, Vigano D, Realini N, Guidali C, Braida D, Capurro V, et al. Chronic delta 9-tetrahydrocannabinol during adolescence provokes sex-dependent changes in the emotional profile in adult rats: behavioral and biochemical correlates. Neuropsychopharmacology. 2008;33:2760-71.

17. Wang G, Coleman CG, Chan J, Faraco G, Marques-Lopes J, Milner TA, et al. Angiotensin II slow-pressor hypertension enhances NMDA currents and NOX2dependent superoxide production in hypothalamic paraventricular neurons. Am J Physiol Regul, Integr Comp Physiol. 2013;304:R1096-106.

18. Degenetais E, Thierry AM, Glowinski J, Gioanni Y. Electrophysiological properties of pyramidal neurons in the rat prefrontal cortex: an in vivo intracellular recording study. Cereb Cortex. 2002;12:1-16.

19. Paxinos G, Franklin K. The mouse brain in stereotaxic coordinates. San Diego: Academic Press; 2012

20. Milner TA, Waters EM, Robinson DC, Pierce JP. Degenerating processes identified by electron microscopic immunocytochemical methods. Methods Mol Biol. 2011;793:23-59.

21. Chan J, Aoki C, Pickel VM. Optimization of differential immunogold-silver and peroxidase labeling with maintenance of ultrastructure in brain sections before plastic embedding. J Neurosci Methods. 1990;33:113-27.

22. Frederick AL, Saborido TP, Stanwood GD. Neurobehavioral phenotyping of $G$ (alphaq) knockout mice reveals impairments in motor functions and spatial working memory without changes in anxiety or behavioral despair. Front Behav Neurosci. 2012;6:29.

23. Glass MJ, Robinson DC, Waters E, Pickel VM. Deletion of the NMDA-NR1 receptor subunit gene in the mouse nucleus accumbens attenuates apomorphineinduced dopamine D1 receptor trafficking and acoustic startle behavior. Synapse. 2013;67:265-79

24. Fitzgerald ML, Lupica CR, Pickel VM. Decreased parvalbumin immunoreactivity in the cortex and striatum of mice lacking the CB1 receptor. Synapse. 2011;65:827-31.
25. Peters A, Palay S, Webster H. The fine structure of the nervous system. New York: Oxford University Press; 1991.

26. Stanwood GD, Parlaman JP, Levitt P. Anatomical abnormalities in dopaminoceptive regions of the cerebral cortex of dopamine D1 receptor mutant mice. J Comp Neurol. 2005;487:270-82.

27. Crawley JN. Designing mouse behavioral tasks relevant to autistic-like behaviors. Ment Retard Dev Disabil Res Rev. 2004;10:248-58.

28. Thompson BL, Levitt P, Stanwood GD. Prenatal cocaine exposure specifically alters spontaneous alternation behavior. Behav Brain Res. 2005;164:107-16.

29. Papouin T, Ladepeche L, Ruel J, Sacchi S, Labasque M, Hanini M, et al. Synaptic and extrasynaptic NMDA receptors are gated by different endogenous coagonists. Cell. 2012;150:633-46.

30. Katona I, Sperlagh B, Sik A, Kafalvi A, Vizi ES, Mackie K, et al. Presynaptically located $C B 1$ cannabinoid receptors regulate GABA release from axon terminals of specific hippocampal interneurons. J Neurosci. 1999;19:4544-58.

31. Conklin HM, Luciana M, Hooper CJ, Yarger RS. Working memory performance in typically developing children and adolescents: behavioral evidence of protracted frontal lobe development. Dev Neuropsychol. 2007;31:103-28.

32. Bouvier G, Bidoret C, Casado M, Paoletti P. Presynaptic NMDA receptors: roles and rules. Neuroscience. 2015:311:322-40.

33. Larsen RS, Sjostrom PJ. Synapse-type-specific plasticity in local circuits. Curr Opin Neurobiol. 2015:35:127-35.

34. Armbruster M, Hanson E, Dulla CG. Glutamate clearance is locally modulated by presynaptic neuronal activity in the cerebral cortex. J Neurosci. 2016;36:10404-15.

35. Keimpema E, Barabas K, Morozov YM, Tortoriello G, Torii M, Cameron G, et al. Differential subcellular recruitment of monoacylglycerol lipase generates spatial specificity of 2-arachidonoyl glycerol signaling during axonal pathfinding. Neurosci. 2010;30:13992-4007.

36. Mathew SS, Hablitz JJ. Presynaptic NMDA receptors mediate IPSC potentiation at GABAergic synapses in developing rat neocortex. PLoS One. 2011;6:e17311.

37. Dore K, Stein IS, Brock JA, Castillo PE, Zito K, Sjöström PJ. Unconventional NMDA receptor signaling. J Neurosci. 2017;37:10800-07.

38. Häring M, Guggenhuber S, Lutz B. Neuronal populations mediating the effects of endocannabinoids on stress and emotionality. Neuroscience. 2012;204:145-58

39. Fitzgerald $\mathrm{ML}$, Pickel VM. Adolescent isolation rearing produces a prepulse inhibition deficit correlated with expression of the NMDA GluN1 subunit in the nucleus accumbens. Brain Struct Funct. 2018;223:3169-81.

40. Lichnerova K, Kaniakova M, Park SP, Skrenkova K, Wang YX, Petralia RS, et al. Two $\mathrm{N}$-glycosylation sites in the GluN1 subunit are essential for releasing N-methyl-daspartate (NMDA) receptors from the endoplasmic reticulum. J Biol Chem 2015;290:18379-90

41. Wu Y, Whiteus C, Xu CS, Hayworth KJ, Weinberg RJ, Hess HF, et al. Contacts between the endoplasmic reticulum and other membranes in neurons. Proc Nat Acad Sci USA. 2017;114:E4859-E67.

42. Fukumitsu K, Fujishima K, Yoshimura A, Wu YK, Heuser J, Kengaku M. Synergistic action of dendritic mitochondria and creatine kinase maintains ATP homeostasis and actin dynamics in growing neuronal dendrites. J Neurosci. 2015;35:5707-23.

43. Montgomery JM, Selcher JC, Hanson JE, Madison DV. Dynamin-dependent NMDAR endocytosis during LTD and its dependence on synaptic state. BMC Neurosci. 2005;6:48.

44. Harsing LG, Zsilla G, Matyus P, Nagy KM, Marko B, Gyarmati Z, et al. Interactions between glycine transporter type 1 (GlyT-1) and some inhibitor molecules-glycine transporter type 1 and its inhibitors (review). Acta Physiol Hung. 2012;99:1-17.

45. Finlay JM, Dunham GA, Isherwood AM, Newton CJ, Nguyen TV, Reppar PC, et al. Effects of prefrontal cortex and hippocampal NMDA NR1-subunit deletion on complex cognitive and social behaviors. Brain Res. 2015;1600:70-83.

46. You IJ, Jung YH, Kim MJ, Kwon SH, Hong SI, Lee SY, et al. Alterations in the emotional and memory behavioral phenotypes of transient receptor potential vanilloid type 1-deficient mice are mediated by changes in expression of 5-HT(1) A, GABA(A), and NMDA receptors. Neuropharmacology. 2012;62:1034-43.

47. Deacon RM, Penny C, Rawlins JN. Effects of medial prefrontal cortex cytotoxic lesions in mice. Behav Brain Res. 2003:139:139-55.

48. Chan T, Kyere K, Davis BR, Shemyakin A, Kabitzke PA, Shair HN, et al. The role of the medial prefrontal cortex in innate fear regulation in infants, juveniles, and adolescents. J Neurosci. 2011;31:4991-99. 